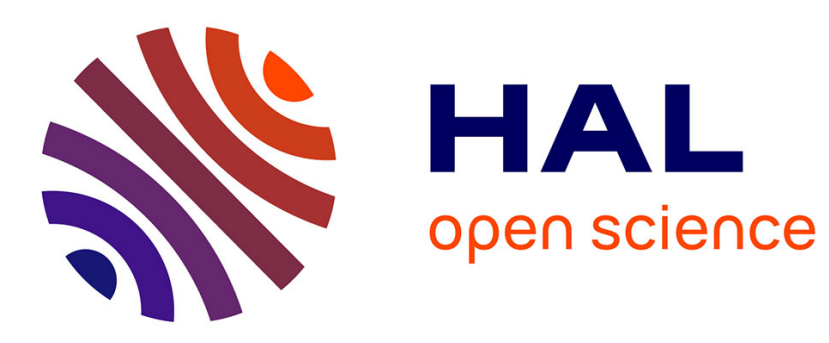

\title{
A new global approach to design for additive manufacturing
}

Rémi Ponche, Jean-Yves Hascoët, Olivier Kerbrat, Pascal Mognol

\section{To cite this version:}

Rémi Ponche, Jean-Yves Hascoët, Olivier Kerbrat, Pascal Mognol. A new global approach to design for additive manufacturing. Virtual and Physical Prototyping, 2012, 7 (2), pp.93-105. 10.1080/17452759.2012.679499 . hal-00781211

\section{HAL Id: hal-00781211 \\ https://hal.science/hal-00781211}

Submitted on 17 Feb 2013

HAL is a multi-disciplinary open access archive for the deposit and dissemination of scientific research documents, whether they are published or not. The documents may come from teaching and research institutions in France or abroad, or from public or private research centers.
L'archive ouverte pluridisciplinaire HAL, est destinée au dépôt et à la diffusion de documents scientifiques de niveau recherche, publiés ou non, émanant des établissements d'enseignement et de recherche français ou étrangers, des laboratoires publics ou privés. 


\title{
A new global approach to design for additive manufacturing R. PONCHE ${ }^{1}$ J.Y. HASCOET, O. KERBRAT, P. MOGNOL
}

Institut de Recherche en Communications et Cybernetique de Nantes (UMR CNRS 6597), Nantes, France

\begin{abstract}
:
Nowadays, due to rapid prototyping processes improvements, a functional part can be built directly through additive manufacturing. It is now accepted that these new processes can increase productivity while enabling a mass and cost reduction and an increase of the parts functionality. However, in order to achieve this, new design methods have to be developed to take into account the specificities of these processes, with the Design For Additive Manufacturing (DFAM) concept. In this context, a methodology to obtain a suitable design of parts built through additive manufacturing is proposed; both design requirements and manufacturing constraints are taken into account.
\end{abstract}

\section{Introduction}

Recent progress has permitted transition from Rapid Prototyping to Additive Manufacturing $(\mathrm{AM})$. Indeed, today, with this kind of manufacturing process, not only prototypes can be produced but also real functional parts in current materials including metals, polymers and ceramics [1]. Because AM for production eliminates the need of tooling and can generate free forms, many of the current restrictions of design for manufacturing and assembly are no longer valid [2]. However, whatever the technology used [3], as in all the manufacturing processes, the $\mathrm{AM}$ ones have characteristics and specificities of their own which may have an impact on the manufactured parts quality. In order to utilize in the best way the AM possibilities in terms of design and to ensure the quality of the produced parts, a global numerical chain which allows to move from functional specifications of a part to its manufacturing, must be defined (figure 1). The purpose of this numerical chain [4] is to reach a global process control from knowledge of process obtained from experimentations, measurements and simulations. Among the prerequisite to achieving such numerical chain, a Design For Manufacturing (DFM) [5] approach is required which allows the taking into account of the AM processes capabilities and limits directly from the design stage.

\subsection{Design for additive manufacturing}

Several works have been carried out concerning the classical DFM approach [6] for AM. Concerning manufacturability estimation, manufacturing cost and time have been analysed $[7,8]$ according to the manufacturing sequence. Similarly, the relationship between parts surfaces quality and manufacturing sequence has been studied [9, 10]. From these different specific works, [11] proposed a methodology to map parts in relation to its manufacturability. Based only on geometrical analysis, these studies are limited because they do not take into account the physical phenomena which occur during the manufacturing process $[12,13]$ which may have an impact on parts quality [14] and therefore on their manufacturing time and cost. Concerning manufacturability improvement, there have been very few studies reported on AM processes. General build guidelines have been established [15, 16] and a methodology proposed by [17] enables modification of some non-critical geometric features. Moreover, a method for providing mesostructures

1. Corresponding author. Email : Remi.Ponche@irccyn.ec-nantes.fr, Jean-Yves.Hascoet@irccyn.ec-nantes.fr, Pascal.Mognol@irccyn.ec-nantes.fr, Olvier.kerbrat@irccyn.ec-nantes.fr 


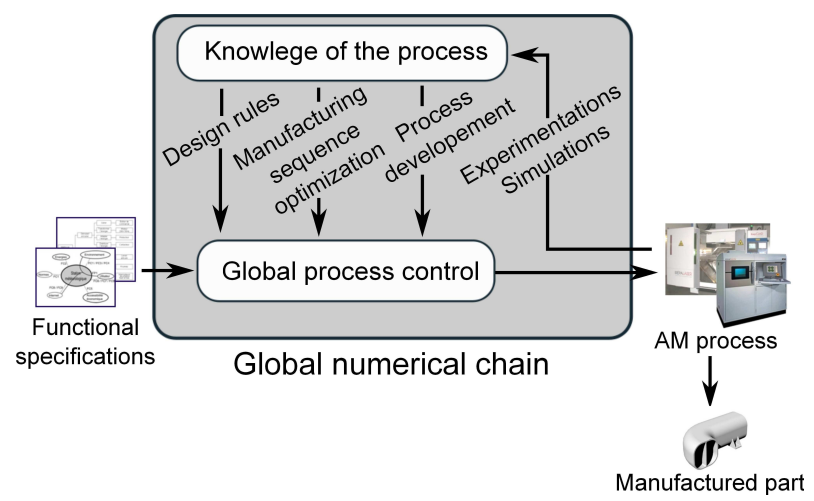

FigURE 1 - Global numerical chain concept.

within a part so as to achieve improved functional requirements part has been established [18]. Here again, the purpose is to minimize fabrication cost and time without a real awareness of the process planning, which do not allow to guarantee the parts quality expected. In addition to these limitations, classical DFM approaches may restrict the new perspectives of design opened up by the AM processes.

\subsection{Partial approach versus global approach}

Indeed, all these works enable to determine and to improve the manufacturability of a part from its CAD model for a given AM process. Because they start from an initial geometry (given by the initial CAD model), these analysis can be qualified as "partial approaches" (figure 2). In this case, it is difficult to determine the real optimized characteristics for a given AM process while fulfilling original functional specifications. Indeed, the initial CAD model was thought to be manufactured by an initial manufacturing process often very different from an AM process (for example machining which is the most often used), moreover the proposed modifications are local and the result is never far from the initial design. The CAD model that is obtained is thus never really designed for the AM process that is chosen. On the contrary, a "global approach" (figure 3) starts directly from both the chosen manufacturing process characteristics and the functional specifications of the parts to design. Designers can thus determine the geometry which optimizes the use of the chosen AM process characteristics while meeting the functional specifications. The purpose is not to limit geometry by an initial idea of the part shapes but to define it only from the manufacturing process and the functional specifications. This new way of thinking is in opposite with traditional Design For Manufacturing (DFM) methodologies. However, capitalization of the entire knowledge about the manufacturing processes is needed. A beginning of a global approach, based on topology optimization with manufacturing constraints, has been applied to casting process ([19]).

\subsection{Scope of the paper}

Although the global approach seems very interesting, no existing work has been carried out in this way in AM. Because these processes are quite young, still little known and very different from the other manufacturing processes, the psychological inertia phenomena may prevent the designer from utilizing in the best way all their capabilities ([20]). 


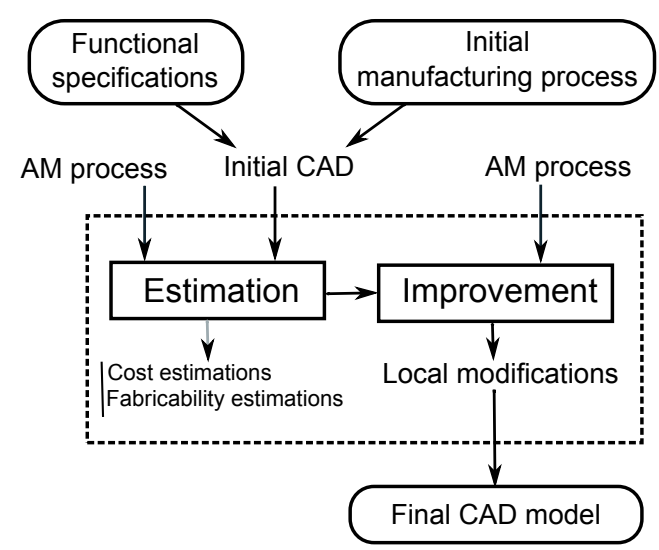

Figure 2 - Partial approach.

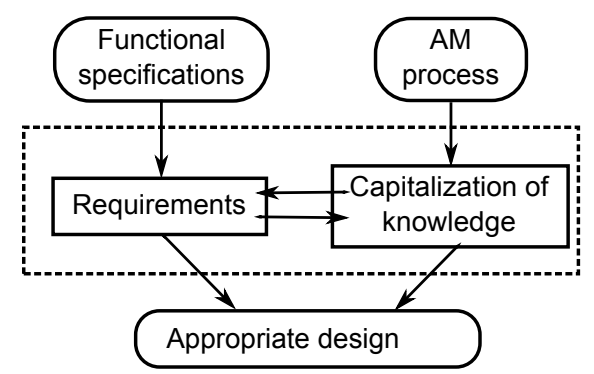

FIGURE 3 - Global approach.

Moreover, most of the AM processes are based on a layer by layer manufacturing where the material is locally merged thanks to a local moving energy source (usually a laser or an electron bean) which follows a programmed manufacturing trajectory. It entails that the characteristics of the manufactured volumes, in terms of microstructure [21], geometry [14] and manufacturing time [10] depend first on the manufacturing direction and secondly on the manufacturing trajectories. In addition to the consideration of the processes' characteristics, the choice of the Manufacturing Direction $(M D)$ and the Manufacturing Trajectories $(M T)$ according to functional specifications is thus the key of a global DFAM which would facilitate designers to explore new design spaces. That is why a new methodology which starts directly from both the functional specifications and the process characteristics is proposed in this paper.

Firstly, in section two, the required data is presented. Then in section three, the different steps of the methodology, based on the choice of $M D$, are explained. The methodology has been applied on a part manufactured by a Powder Based Metal Deposition (PBMD) process [22] ; this constitutes the fourth section.

\section{Proposed requirements for a global DFAM}

In this section, requirements for a global DFAM are presented. They constitute the required data for the proposed methodology. 


\subsection{Functional specifications}

The global purpose is to propose a structured approach which would help the designer to integrate the knowledge of the chosen AM process in his design to meet the functional specifications. The functional specifications can be detailed as :

- Functional surfaces $(F S)$ : type, dimensions and position

- Dimensional and geometrical specifications, linked to the FS

- Mechanical requirements, they depend on the chosen material characteristics

- "Empty volumes" : dimensions and position. They correspond to the volumes which must not contain material, due to the assembly constraints of the designed part into the system to which it belongs.

\subsection{Context}

The study context is an influential factor. Because it can be translated into a concrete objective in terms of mass, cost or manufacturing time, it has thus to be taken into account too.

\subsection{Manufacturing characteristics}

Manufacturing characteristics are linked to one another and they cannot be seen separately, a global view which draws upon all the knowledge and experience of the community is consequently needed. The main characteristics of the manufacturing machines which must be taken into account are :

- Kinematics

- Maximal and minimal dimensions

- Capability in terms of accuracy

- Required accessibility

But also physical phenomena involved in the manufacturing process, which are decisive in terms of final properties of parts and which are linked to the manufacturing sequence.

\subsection{Finishing process characteristics}

Similarly, if specifications (geometrical and dimensional) cannot be directly reached by the AM process that is chosen, a finishing process is needed. Because it can influence the final geometry, in particular in terms of overthickness and required accessibility, it has to be taken into account.

\section{The proposed design methodology}

From this data, a structured methodology can help designers in taking into account the manufacturing constraints while suggesting him an appropriate design for AM. The methodology is presented in figure 4. It is divided into three main steps which enable to include gradually manufacturing knowledge in the shapes and the volumes of the parts to be designed. The first step is a global analysis which allows to delimit the design problem in terms of geometrical dimensions in relation to the dimensional characteristics of the AM process. The second one allows to fulfil the dimensional and geometrical specifications in relation to the AM process capability and the finishing process characteristics. Finally, the third step allows to fulfil the physical and assembly requirements in relation to the capability of the AM process 


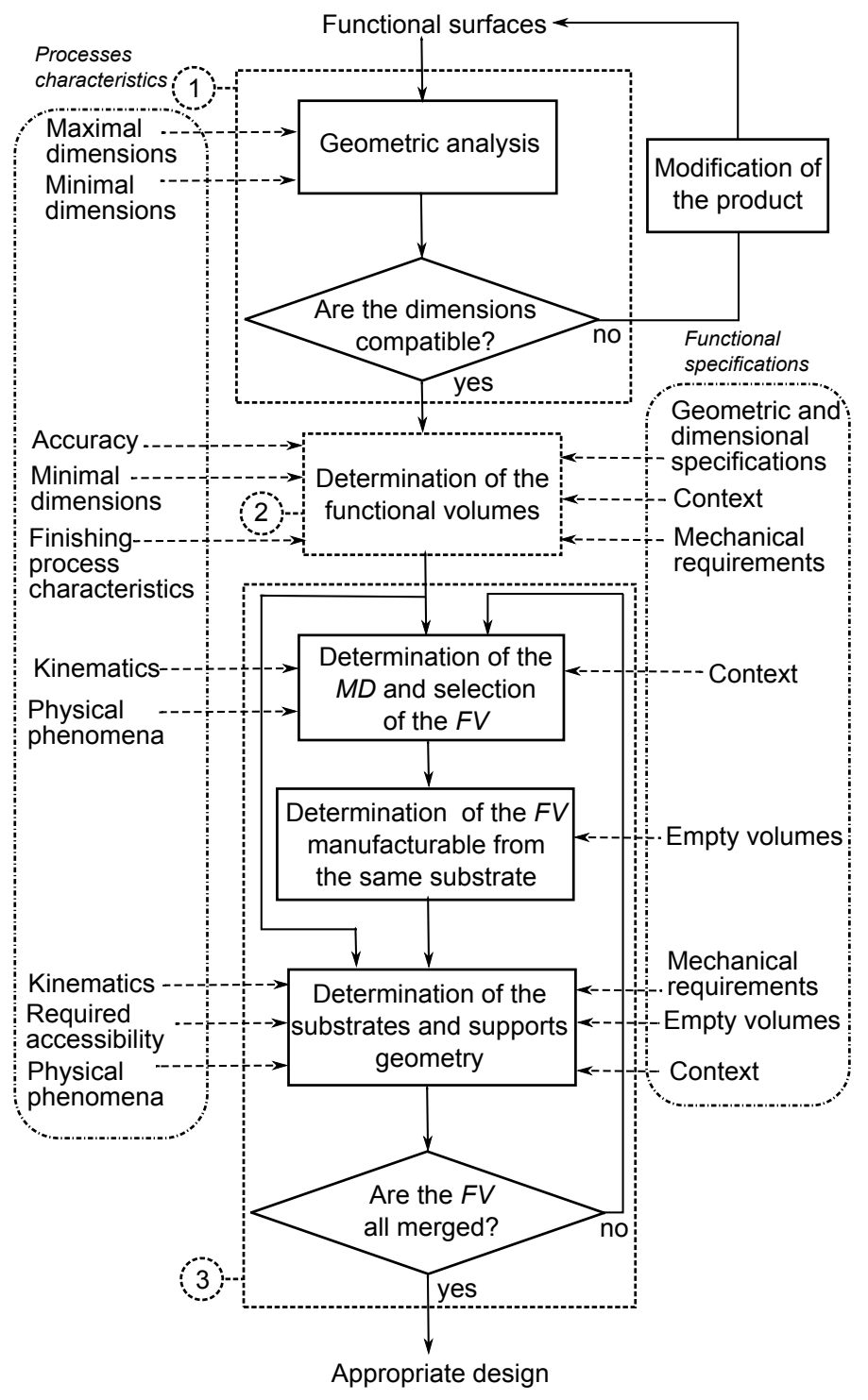

FIGURE 4 - The proposed DFAM methodology.

\subsection{Step 1 : Analysis}

The $F S$ are obtained from the functional analysis of the product and are given by the designer. The first methodology step enables to find out if all the surfaces can be merged with one another by the chosen AM process into a single part. A first geometrical analysis is carried out; it takes into account the maximal and minimal dimensions which can be obtained by the chosen manufacturing machine. If the dimensions are not suitable, the product has to be modified or divided into different parts by the designer and the functional specifications of these new parts are then studied. 


\subsection{Step 2 :Determination of the functional volumes}

The Functional Volumes $(F V)$ are defined directly from the $F S$ on to which a thickness is added. Indeed, only the tolerances in the normal direction of the surfaces are significant in terms of functionality. The others are initially ignored. The thickness, denoted $E$, depends on :

- Dimensional accuracy of the AM process denoted $a$

- Tolerances linked to the $F S$, denoted $p$

For each $F S$, there are two different possibilities : firstly, if a finishing step is not needed $(p \geq a)$, thickness is determined from equation 1 which ensures the functional minimal thickness and from equation 2 which ensures the compatibility with the AM process.

$$
\begin{gathered}
E \geq t+\frac{a}{2} \\
E=n . d-(n-1) . d . \alpha
\end{gathered}
$$

where $t$ is the minimal thickness corresponding to the local mechanical requirements; $d$ is the minimal dimension that can be obtained by the chosen AM process; $\alpha$ is the overlap between two adjacent paths; and $n$ is a positive integer. Equation 1 is illustrated in figure 5(a). If a finishing step is needed $(p<a)$, then the thickness is determined from equations 2,3 and 4 (figure $5(\mathrm{a})$ ).

$$
\begin{aligned}
& E \geq t+\frac{a}{2}+e_{\min } \\
& E \leq t+\frac{a}{2}+e_{\max }
\end{aligned}
$$

Where $e_{\min }$ and $e_{\max }$ are the minimal and maximal overthicknesses which depend on the finishing process and the surfaces geometry. There can be different values of the parameters $d$, $\alpha$ and $a$ (according to the $M D)$.

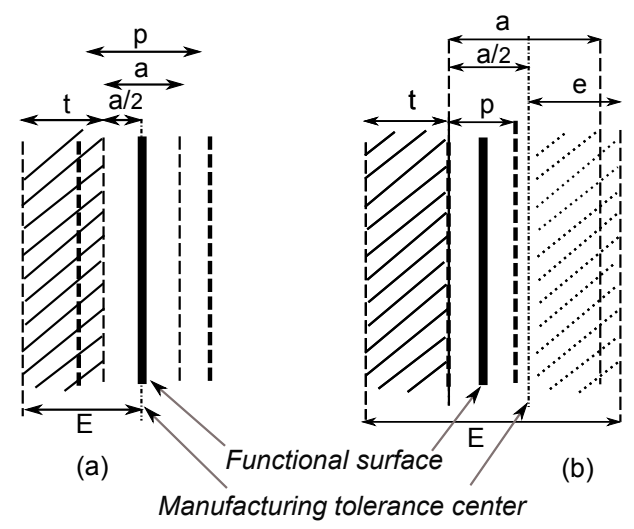

Figure 5 - Definitions of the thickness : (a) without finishing(b) with finishing.

\subsection{Step 3 : Determination of the linking volumes}

The purpose of this step is to merge the $F V$ to define the volumes of the part while taking into account at best both design requirements and manufacturing constraints. In the case of additive manufacturing, volumes are usually obtained layer by layer. It involves that their geometries 
strongly depend on the $M D$. Indeed, the choices of the $M D$ have a direct influence on material quantity (need of supports), build time [23] and mechanical properties [24]. Moreover, the $M T$ defining the energy source path during the process, have a strong impact on the physical phenomena that occur during the manufacturing process and therefore on the final part quality [25]. That is why, the determination of the $M D$ and of the $M T$ is at the center of the methodology.

The Linking Volume $(L V)$ definition is divided into four steps. The first step is to determine the most critical $M D$, which can be characterized by the shape or the number of $F V$ which can be manufactured in the same way. It is carried out according to the study context and the capability of the process given by its kinematics and the physical phenomena involved. The second step is, in the chosen $M D$, to merge the selected $F V$ which can be manufactured from the same substrate. It is carried out according to the empty volumes, which must not be, in the $M D$, between two $F V$ manufactured from a same substrate. In the third step, the substrates and supports shapes are determined according to :

- The selected $F V$

- The other $F V$

- The kinematics of the process

- The accessibility required by the process

- The mechanical requirements

- The empty volumes

- The study context

- The physical phenomena that occur during the process

The latter being strongly linked to the type and the shape of the $M T$ that are used, it is essential to select suitable $M T$ among all the possibilities to control them and to guaranty the expected geometrical quality. A classification of the different $M T$ that are possible has been done (figure 6) to describe and parametrize each one of them.

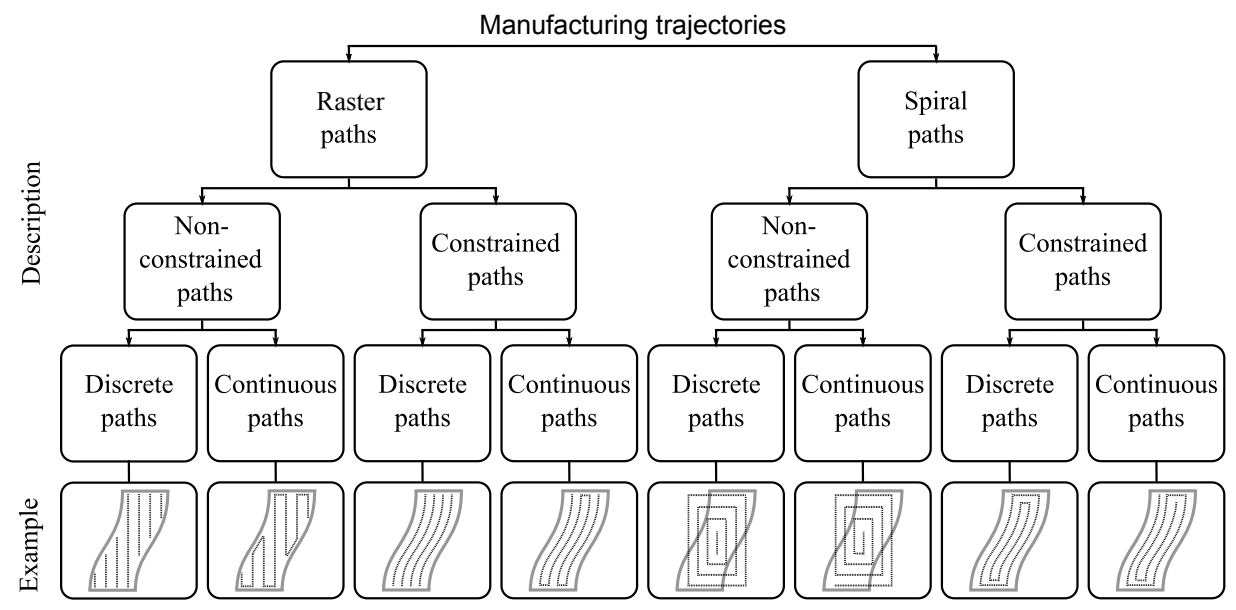

Figure 6 - Classification of the Manufacturing Trajectories $(M T)$.

The last step is to check if all the volumes are merged. If it is not the case, the first three steps are repeated while taking into account the $F S$ that have not been yet analyzed and the substrates obtained previously and so on until all the $F V$ are merged. In the end, the process is complete and an appropriate design for the chosen AM process is obtained. Indeed, the AM process specifications are taken into account step by step in parallel with the functional requirements. 
this ensures that the most possible process-related knowledge is taken into account to obtain the final shapes of the studied part.

\section{Example}

The proposed methodology has been applied to a case of a robot hinge in stainless steel (figure 7). The study input data are detailed then the three steps of the DFAM methodology are illustrate in this section.

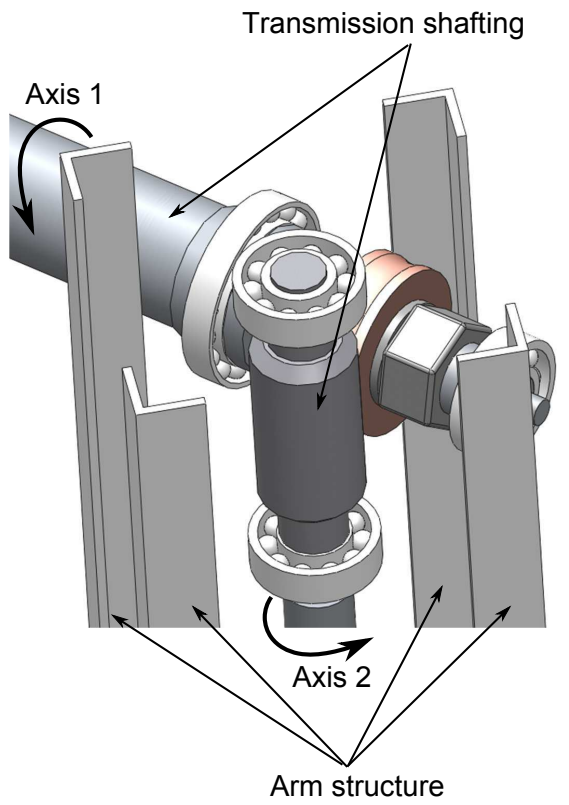

FiguRE 7 - Global view of the studied system.

\subsection{Input data}

\subsubsection{Functional specifications}

The case is composed of twenty FS : four bearing holders (hollow cylinders) and sixteen flat surfaces, which are shown with their nominal dimensions in black in figure 8 . To enable the assembly of the case with the other parts of the robot, some empty volumes are defined. They are represented by the transparent volumes.

The functional analysis of the robot has enabled to determine the geometrical and dimensional specifications linked with each surfaces. An extract is shown in figure 9. The mechanical requirements are translated into a final minimal thickness $t$ for each surface. It is $5 \mathrm{~mm}$ for the hollow cylinders and $3 \mathrm{~mm}$ for the based planes.

\subsubsection{Context}

Because of the robotic context, the global objective is to minimize the mass of the studied part. 


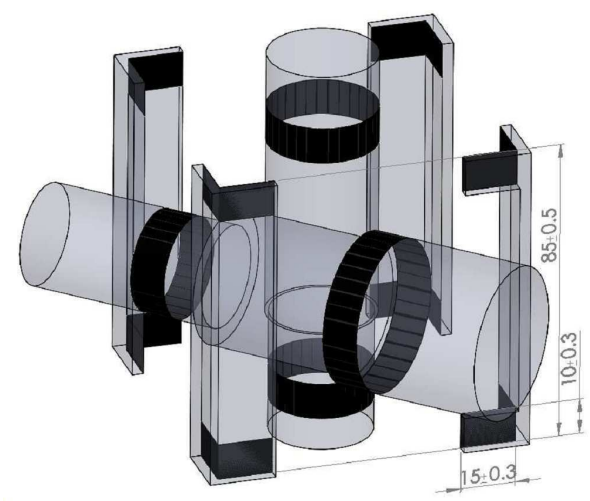

Figure 8 - The $F S$ and the empty volumes of the part to design.

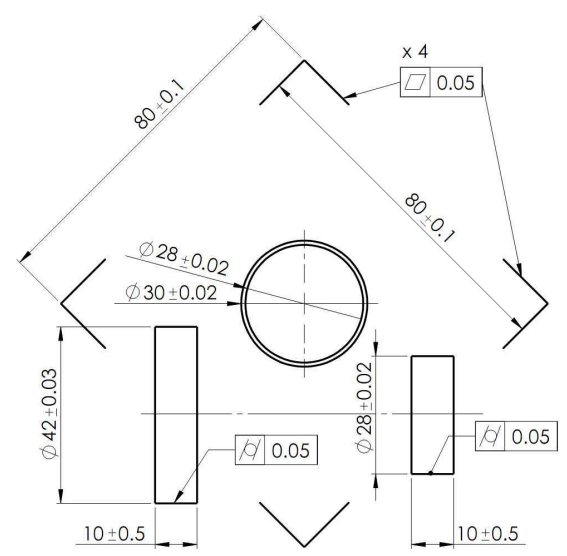

FigURE 9 - An extract of the specifications taken from the functional analysis of the case.

\subsection{Manufacturing characteristics}

\subsubsection{The additive manufacturing process}

The AM process chosen to manufacture this case is the CLAD process (figure 10). It is a PBMD process, based on the 3D layer by layer deposition of laser melted powders. Its main characteristics are presented in table 1.

In this example, the assumptions that parameters $d, a$ and $\alpha$ are equal in all the directions is made.

The constraints due to the physical phenomena linked with the process (in particular the thermal phenomena) are considered in ways : first, the substrates thickness must be at least equal to the thicknesses of the volumes that it enables to manufacture. Secondly, because the discontinuities in the $M T$ may generate an unwanted variation of the process parameters and 


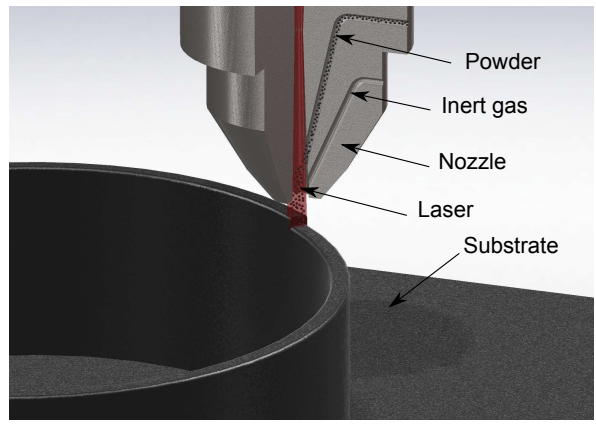

Figure 10 - Clad process.

$\begin{array}{lccccc}\text { Kinematics } & \begin{array}{c}\text { Required accessibility } \\ (\mathrm{mm})\end{array} & \begin{array}{c}\text { Maximal dimensions } \\ (\mathrm{mm})\end{array} & \begin{array}{c}\alpha \\ (\mathrm{mm})\end{array} & \begin{array}{c}\alpha \\ (\mathrm{mm})\end{array} & \begin{array}{c}a \\ (\mathrm{~mm})\end{array} \\ 5 \text { axis } & \emptyset 60 & 500^{*} 560^{*} 700 & 0.8 & 0.3 & 0.2\end{array}$

TABLE 1 - CLAD process characteristics.

also a gap between the designed and the manufactured part, the geometry will be chosen to limit them.

\subsubsection{The finishing process}

The finishing process that is chosen is high speed milling; in view the specification related to the $F S$, this choice involves a minimal and a maximal overthickness of $0.5 \mathrm{~mm}$ and $1 \mathrm{~mm}$ respectively.

\subsection{Step 1 : Analysis}

According to the geometrical analysis of the functional surfaces (figures 8 and 9) and to the maximal and minimal dimensions imposed by the manufacturing process (table 1), all the dimensions are compatible and all the $F S$ can be merged in only one part.

\subsection{Step 2 : determination of the functional volumes}

The thicknesses $E$ are determined from the different parameters of equations 2 and 3 ; For each $F S$ the results are given in table 2. All the functional volumes are shown in figure 11(a).

$\begin{array}{lcccccc}\text { Surface type } & t & a & e_{\min } & e_{\max } & d & E \\ (\mathrm{~mm}) & (\mathrm{mm}) & (\mathrm{mm}) & (\mathrm{mm}) & (\mathrm{mm}) & (\mathrm{mm}) \\ \text { Cylinder } & 5 & 0.2 & 0.5 & 1 & 0.8 & 5.9 \\ \text { Flat } & 3 & 0.2 & 0.5 & 1 & 0.8 & 3.6\end{array}$

TABLE 2 - Definition of the $F S$ thicknesses. 


\subsection{Step 3 : determination of the linking volumes}

The $(M D)$ are determined, due to the robotic context, to minimize the mass of the part and therefore the support structures quantity. A geometrical analysis of the $F V$ enables to determine two $M D: Z_{1}$ and $Z_{2}$ (figure 11(a)). $Z_{1}$ enables to manufacture heighten $F V$ (in dark grey) and $Z_{2}$ enables to obtain the two last (in light grey). If one or other of the $M D$ is favoured (which means that it is analysed in first), the final part geometry could be not the same. Both cases will be therefore analysed and the geometry which represented the best way of satisfying the study context will be selected. In case $1, Z_{1}$ is favoured whereas in case $2, Z_{2}$ is favoured.

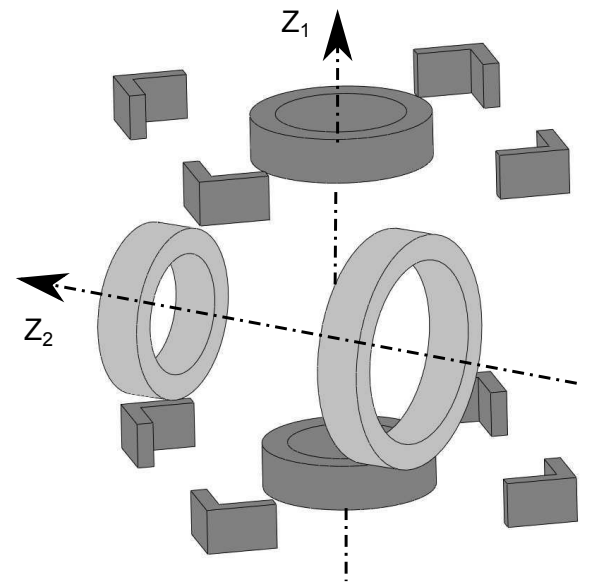

(a) The $F V$.

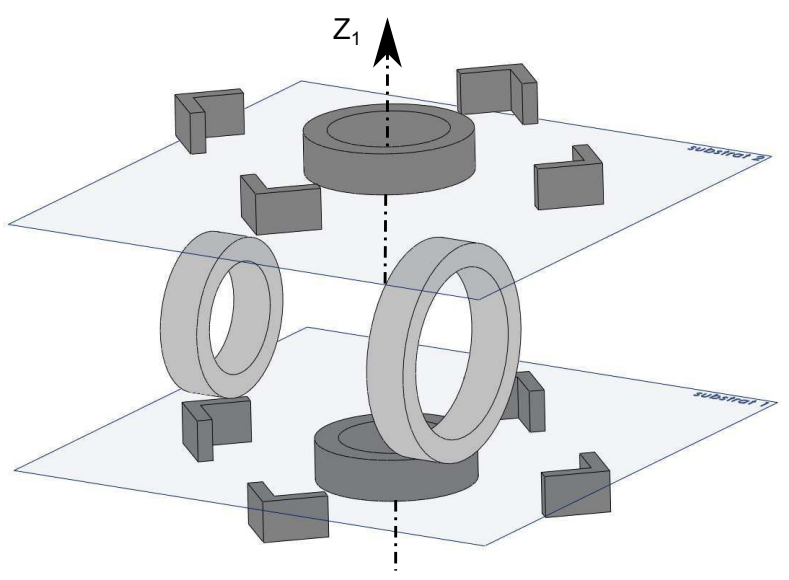

(b) Position of the substrates.

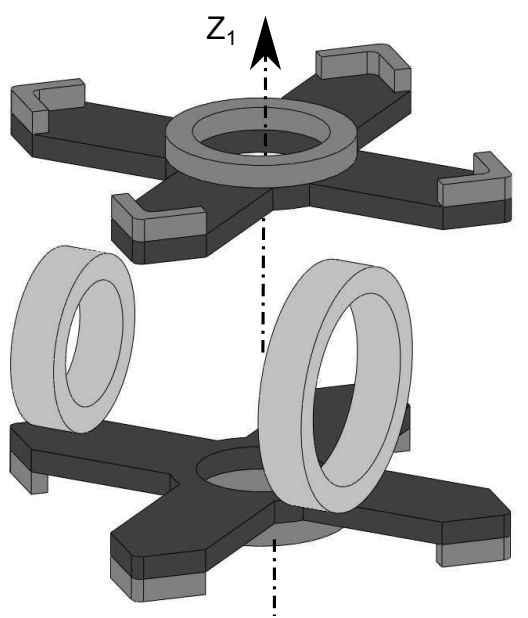

(c) The $L V$ geometry in $Z_{1}$.

Figure 11 - Definition of the $L V$ in $Z_{1}$ when $Z_{1}$ is favoured.

\subsubsection{Case 1 : when $Z_{1}$ is favoured}

Linking volumes in $Z_{1}$ To minimize supports in the empty volumes shown in figure 8 and thus to minimize the finishing operations, all the selected $F V$ linked to $Z_{1}$ must not be manufactured 
together but from two different substrates. Their positions are determined (figure 11(b)) to minimize supports, time of finishing and to guarantee accessibility for the powder feed nozzle.

The selected $F V$ geometry is locally modified in relation to the value of $e_{\max }$ and $e_{\min }$ to minimize the discontinuity of the $M T$. In particular here, in the case of the raster discrete paths, it involves to define a radius $R$ (figure 12) which value is given by equation 5 .

$$
R=\frac{\sqrt{(2)}}{\sqrt{(2)-1}} \cdot\left(e_{\max }-e_{\min }\right)
$$

Then the geometry of the substrates is defined according to the $F V$ position and their thickness (in black in figure 11(c)). Because all $F V$ are not merged, a second $M D$ is analysed.

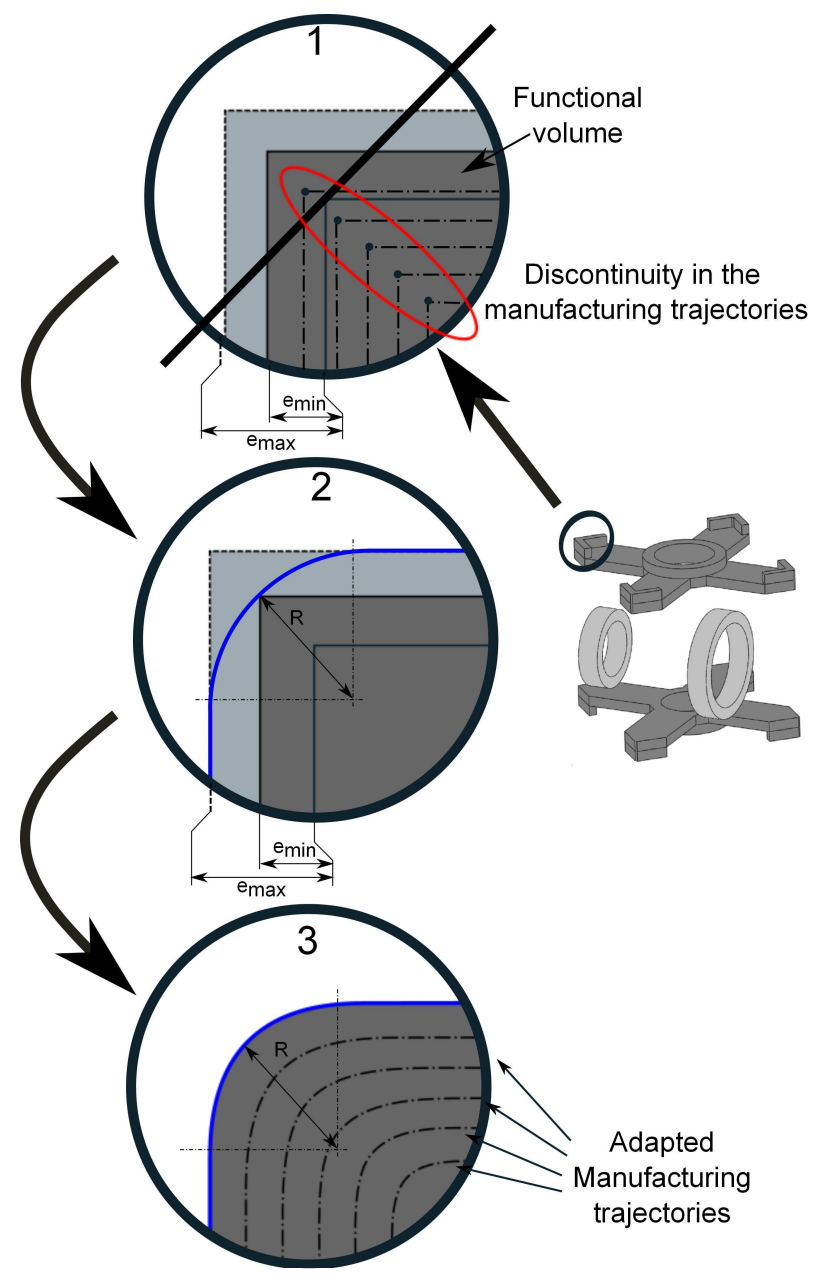

FiguRE 12 - Local geometrical modifications to avoid the $M T$ discontinuities.

Linking volumes in $Z_{2}$ As previously, because of the empty volumes, the two cylinders linked to $Z_{2}$ cannot be manufactured together. It involves two substrates (figure 13(a)). Moreover, the space between these $F V$ and those already analysed (those linked to $Z_{1 \text { ) }}$ ) being lower than 
the required one by the powder feed nozzle of the CLAD machine (table 1), the accessibility requirements is not satisfied and the $M D Z_{2}$ cannot be used, the process is repeated one more time.

Linking volumes in $Z_{3}$ A third $M D Z_{3}$ is determined from the previous substrates (linked to $Z_{1}$ ) and $F V$ (figure 13(b)). The substrate and supports linked to $Z_{3}$ are determined, as previously, due to the context, to minimize the material quantity and to avoid, as much as possible the empty volumes. The substrate dimensions are given by the volumes that it merges. The supports geometry are simply defined by the orthogonal projection of the volumes linked to $Z_{3}$ onto the substrate (figure 13(c)). Finally, all the $F V$ are merged, the design process is then complete and the final blank part is obtained.

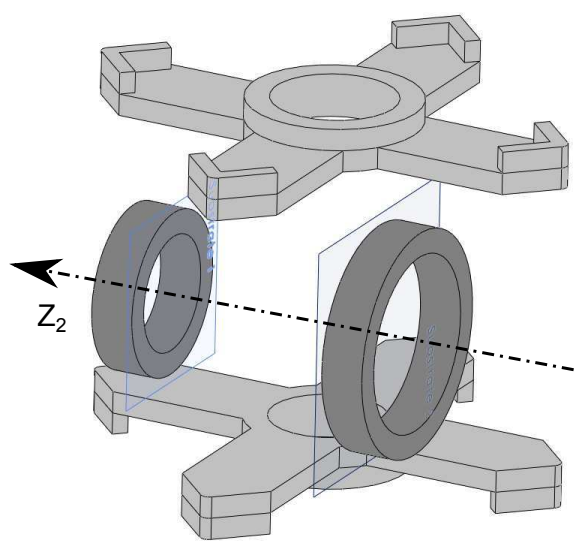

(a) Position of the substrates in $Z_{2}$.

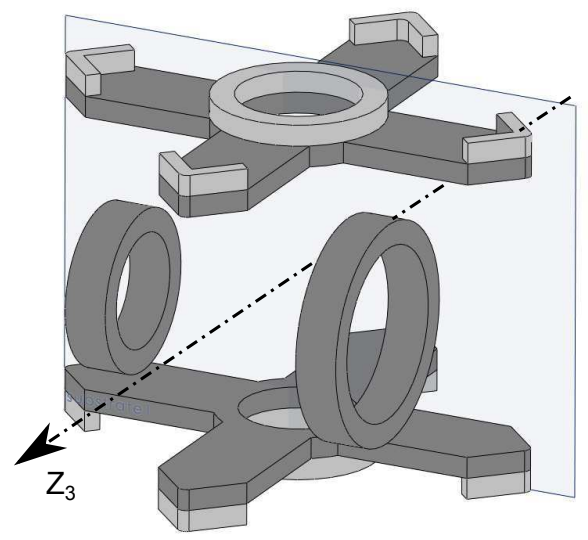

(b) Position of the substrate in $Z_{3}$.

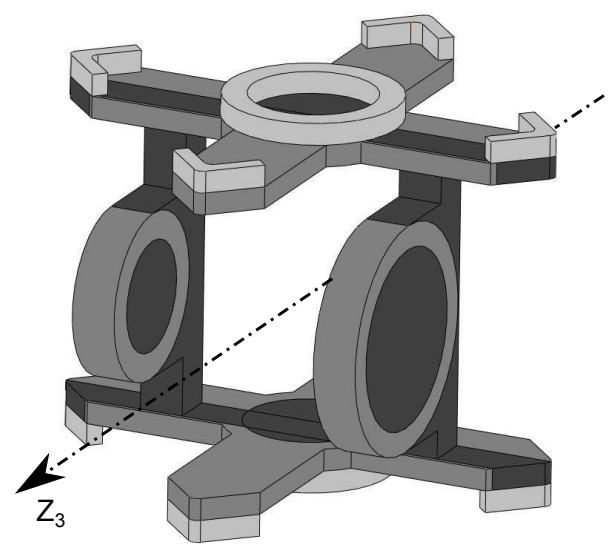

(c) Geometry of the substrate and the supports in $Z_{3}$.

Figure 13 - Definition of the $L V$ in $Z_{2}$ then in $Z_{3}$ when $Z_{1}$ is favoured. 


\subsubsection{Case 2 : when $Z_{2}$ is favoured}

From the $F V$ in figure 11(a), the same reasoning is applied, starting with the analysed of $Z_{2}$.

Linking volumes in $Z_{2}$ In the same way as above, because of the empty volumes, the two cylinders linked to $Z_{2}$ cannot be manufactured together. Two substrates whose geometry is shown in black in figure 14(a) are therefore defined.

Linking volumes in $Z_{1} \quad$ Similarly, all the $F V$ linked to $Z_{1}$ cannot be manufactured together. First, as in the case where $Z_{1}$ was favoured, to minimize supports in the empty volumes, a minimum of two separated substrates is needed. Moreover, because of the manufacturing of the cylinders linked to $Z_{2}$, the accessibility required by the powder feed nozzle involves that each one of these two substrates have to be subdivided once again into tree separated substrates. In the same way as previously, the geometry of the $F V$ linked to $Z_{1}$ is locally modified in relation to the value of $e_{\max }$ and $e_{\min }$ and then the substrates geometry is defined (in black in the figure $14(\mathrm{~b}))$.

New linking volumes in $Z_{2}$ All the $F V$ being not merged, an other $M D$ should be thus analysed. Because of the accessibility requirement, all the substrates previously obtained cannot be merged to one another following only one $M D . Z_{2}$, being already selected and allowing to merge again several volumes of the part, is re-analysed. The result is shown in figure 14(c).

Linking volumes in $Z_{3} \quad$ Because all the $F V$ are still not merged, the design process is repeated once again. All the previous substrates (defined in $Z_{2}$ and $Z_{1}$ ) can be merged into a third $M D$ : $Z_{3}$. The substrate position and geometry is determined then the supports are defined by the orthogonal projection of the volumes linked to $Z_{3}$ onto the substrate (figure $14(\mathrm{~d})$ ).

Finally, all the $F V$ are merged, the design process is thus complete and the final blank part is obtained.

\subsection{Final result}

Thanks to the proposed methodology, each shape of the part has been designed in order to utilize the CLADő process characteristics and capabilities to fulfil the study functional specifications while taking into account its general context. In the initial stage, from the geometrical analysis of the functional surfaces and according to the objective of minimizing the final part mass, two manufacturing directions have been selected $\left(Z_{1}\right.$ and $\left.Z_{2}\right)$. Because, the favouring of the one or other may impact on the the final part geometry, the two cases have been studied. The final results, obtained after the considering of the finishing operation which enables to meet the required specifications (geometrical and dimensional) and to remove the material in the empty volumes, are shown in figure 15. A FEM analysis has been done to simulate the mechanical behaviour of the proposed designs under the robot normal condition of use. Because, the results are quite close to each other compared with the material limits, they do not really enable to make a choice between both proposed solutions. However, as it is shown in table 3 , the favouring of $Z_{2}$ over $Z_{1}$ results in a lower mass of the blank part (around 19\%) and of the finished part (around $9 \%)$. It allows therefore a lower manufacturing cost and to meet better the global objective given by the robotic context of the study. This solutions is thus finally selected. 


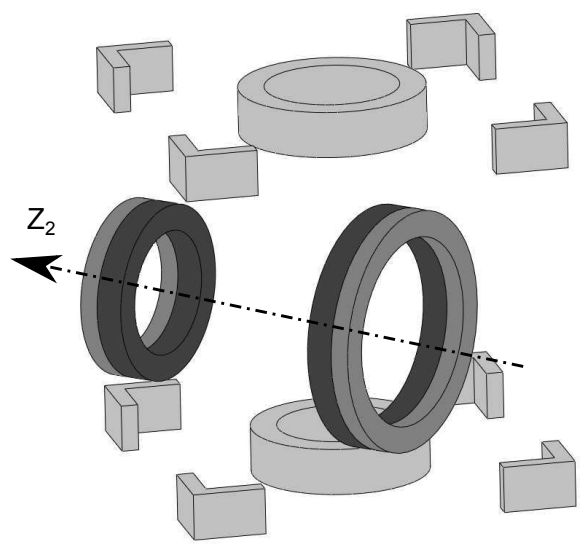

(a) The $L V$ geometry in $Z_{2}$.

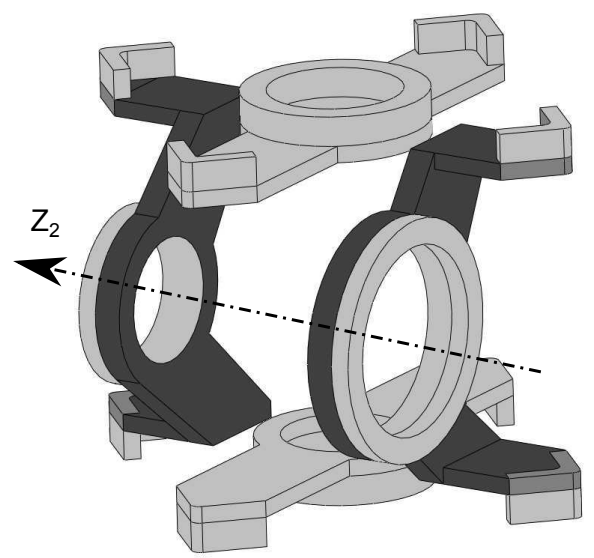

(c) The $L V$ geometry in $Z_{2}$ after its reanalysed.

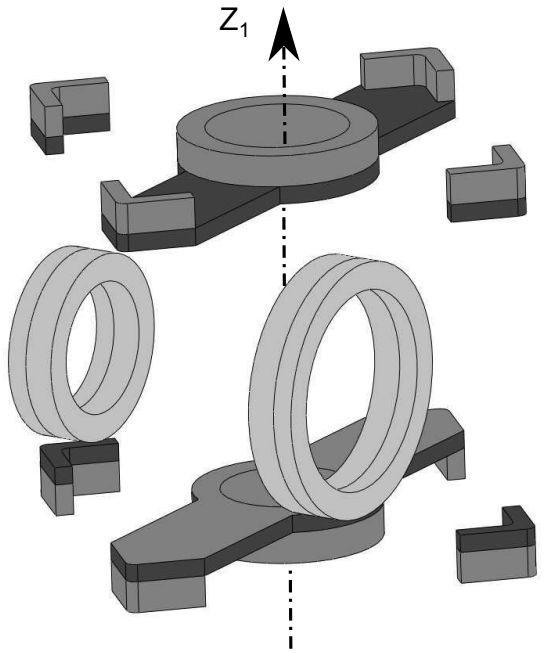

(b) The $L V$ geometry in $Z_{1}$.

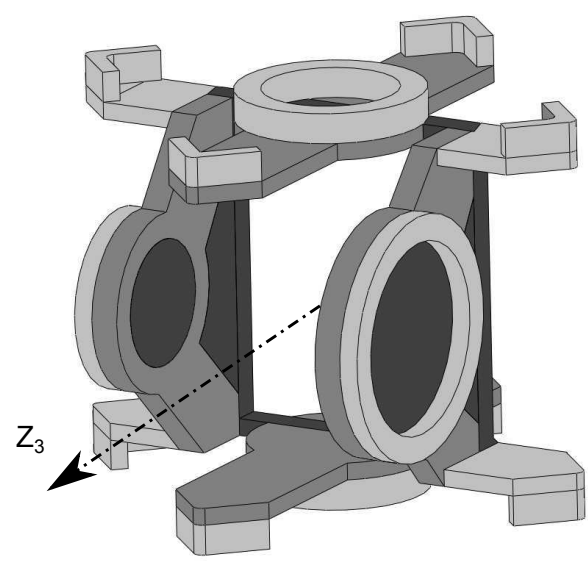

(d) The $L V$ geometry in $Z_{3}$.

Figure 14 - Definition of the different $L V$ when $Z_{2}$ is favoured.

Blank part mass (g)

Finished part mass (g)

Maximal von Mises stress (MPa)

Maximal displacement $(\mathrm{mm})$
When $Z_{1}$ is favoured When $Z_{2}$ is favoured

$\begin{array}{cc}775,9 & 627,5 \\ 548,6 & 499.0 \\ 15,3 & 22,6 \\ 4,110^{-3} & 1,510^{-2}\end{array}$

775,9

27,

22,6

$4,110^{-3} \quad 1,510^{-2}$

TABLE 3 - Characteristics of the two prosed solutions. 


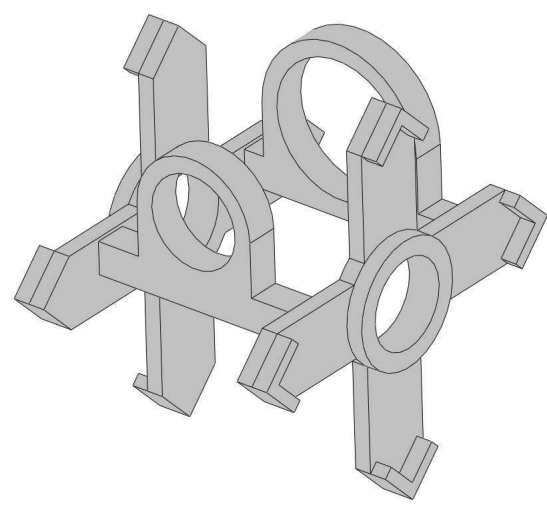

(a) When $Z_{1}$ is favoured.

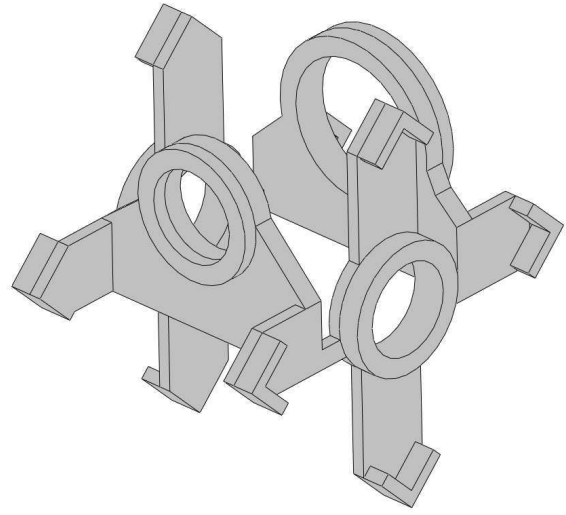

(b) When $Z_{2}$ is favoured.

FiguRE 15 - The final geometry.

\section{Conclusion}

This paper described the initial stage of a promising research project which deals with a global DFAM approach. A new methodology is proposed to obtain an appropriate design for AM processes. Contrary to the classical DFM approaches, to prevent the psychological inertia phenomena which may limit the design innovation and to best utilize the AM processes capabilities, the proposed methodology starts directly from both functional specifications and AM processes characteristics. The required data for a such global DFAM approach has been presented. Then the three steps of the methodology allowing to reach to take into account all of them has been detailed and illustrated by a case study taken from the robotic field.

Further research will be conducted to optimize the methodology in particular regarding the local optimization of the shapes and the internal structures of the linking volumes in terms of functionality as it is done for example by [26]. In parallel, new criteria of choice for manufacturing trajectories will be developed to always more adapt the local geometry regarding to the physical phenomena which occur during the manufacturing process.

\section{Références}

[1] P Mognol, P Muller, and JY Hascoet. A novel approach to produce functionally graded materials for additive manufacturing. In Proceedings of the Conference on Advanced Research in Virtual and Rapid Prototyping, Leiria, Portugal, 2011.

[2] E. Pessard, P. Mognol, J. Y. Hascoët, and C. Gerometta. Complex cast parts with rapid tooling : rapid manufacturing point of view. The International Journal of Advanced Manufacturing Technology, 39(9-10) :898-904, November 2007.

[3] T Wohlers. Wohlers report: Additive Manufacturing State of the Industry. Wohlers associates, Fort Collins, 2010.

[4] Renan Bonnard, Pascal Mognol, and Jean-Yves Hascoët. A new digital chain for additive manufacturing processes. Virtual and Physical Prototyping, 5(2) :75-88, 2010. 
[5] S.K. Gupta, W C Regli, D Das, and D.S. Nau. Automated manufacturability analysis : a survey. Research in Engineering Design, 9(3) :168-190, 1997.

[6] S A Shukor and DA Axinte. Manufacturability analysis system : issues and future trends. International Journal of Production Research, 47(5) :1369-1390, January 2009.

[7] P Alexander. Part orientation and build cost determination in layered manufacturing. Computed-Aided Design, 30(5) :343-356, April 1998.

[8] M. Ruffo, C. Tuck, and R. Hague. Cost estimation for rapid manufacturing laser sintering production for low to medium volumes. Journal of Engineering Manufacture, 220(9) :14171427, January 2006.

[9] Ramakrishna Arni and S.K. Gupta. Manufacturability Analysis of Flatness Tolerances in Solid Freeform Fabrication. Journal of Mechanical Design, 123(1) :148, 2001.

[10] Mircea AncÄČu and Cristian Caizar. The computation of Pareto-optimal set in multicriterial optimization of rapid prototyping processes. Computers 63 Industrial Engineering, 58(4) :696-708, May 2010.

[11] O. Kerbrat, P. Mognol, and J.Y. Hascoet. Manufacturability analysis to combine additive and subtractive processes. Rapid Prototyping Journal, 16(1) :63-72, 2010.

[12] X. He and J. Mazumder. Transport phenomena during direct metal deposition. Journal of Applied Physics, 101(5) :053113, 2007.

[13] S.Y. Wen, Y.C. Shin, J.Y. Murthy, and P.E. Sojka. Modeling of coaxial powder flow for the laser direct deposition process. International Journal of Heat and Mass Transfer, 52(2526) :5867-5877, 2009.

[14] Masoud Alimardani, E Toyserkani, and J Huissoon. A 3D dynamic numerical approach for temperature and thermal stress distributions in multilayer laser solid freeform fabrication process. Optics and Lasers in Engineering, 45(12):1115-1130, December 2007.

[15] Stefano Filippi and Ilaria Cristofolini. The Design Guidelines (DGLs), a knowledge-based system for industrial design developed accordingly to ISO-GPS (Geometrical Product Specifications) concepts. Research in Engineering Design, 18(1) :1-19, April 2007.

[16] G.A. Teitelbaum. Proposed build guidelines for use in fused deposition modeling to reduce build time and material volume. Master of science in mechanical engineering, University of Maryland, 2009.

[17] Shiva Sambu, Yong Chen, and D.W. Rosen. Geometric Tailoring : A Design for Manufacturing Method for Rapid Prototyping and Rapid Tooling. Journal of Mechanical Design, 126(4) :571-580, 2004.

[18] D.W. Rosen. Computer-aided design for additive manufacturing of cellular Structures. Computer-Aided Design $\& 3$ Applications, 4(5) :585-594, 2007.

[19] Lothar Harzheim and Gerhard Graf. A review of optimization of cast parts using topology optimization part II. Structural and Multidisciplinary Optimization, 31(5) :388-399, December 2005.

[20] Richard Hague. Unlocking the Design Potential of Rapid Manufacturing. Rapid manufacturing : an industrial revolution for the digital age, 2006.

[21] L Costa, R Vilar, T Reti, and A Deus. Rapid tooling by laser powder deposition : Process simulation using finite element analysis. Acta Materialia, 53(14) :3987-3999, August 2005.

[22] Jianzhong Ruan, TE Sparks, Z Fan, JK Stroble, and A Panackal. A Review of Layer Based Manufacturing Processes for Metals. In Solid Freeform Fabrication Symposium, pages 233$245,2006$. 
[23] Prabhjot Singh and Debasish Dutta. Multi-Direction Slicing for Layered Manufacturing. Journal of Computing and Information Science in Engineering, 1(2) :129, 2001.

[24] B. Caulfield, P.E. McHugh, and S. Lohfeld. Dependence of mechanical properties of polyamide components on build parameters in the SLS process. Journal of Materials Processing Technology, 182(1-3) :477-488, February 2007.

[25] Ehsan Foroozmehr and R Kovacevic. Effect of path planning on the laser powder deposition process : thermal and structural evaluation. The International Journal of Advanced Manufacturing Technology, 51(5-8):659-669, 2010.

[26] Henrique De Amorim Almeida and Paulo Jorge Da Silva Bártolo. Virtual topological optimisation of scaffolds for rapid prototyping. Medical engineering \& physics, 32(7) :775-82, September 2010. 